\title{
Censura preventiva o repressiva? Note di Pasquale Galluppi sulla legge del 26 luglio 1820 del Regno di Sicilia
}

\section{Preventive or repressive censure? Note from Pasquale Galluppi on the July $26^{\text {th }} 1820$ Act for the Reign of Sicily}

\author{
Maria Concetta BASILE \\ Università di Mesina \\ mcbasile@unime.it
}

Recibido: 19 de mayo de 2016

Aceptado: 23 de junio de 2016

\begin{abstract}
RESUMEN
Se examina en el presente trabajo la difusión y reacciones provocadas por un escrito aparecido en los convulsos años 1820-1821 para el Reino de las Dos Sicilias, en donde se pone de manifiesto la necesidad de la libertad de prensa (el Opuscolo de P. Galluppi es el hilo conductor del trabajo), texto elaborado a propósito de la ley reguladora de esa libertad indispensable para la construcción del Estado liberal y para el surgimiento de la opinión pública al mismo tiempo que dicho escrito activa la labor de la censura en su doble papel de represión y punición, en unos tiempos en donde el Liberalismo no había acabado de asentarse y el Absolutismo se negaba a desaparecer.
\end{abstract}

PALABRAS CLAVE: Nápoles, Sicilia, libertad de expresión, liberta de prensa, censura, opinión pública, Fernando I de Borbón.

\begin{abstract}
The opuscule from P. Galluppi is the leitmotiv of this research, focused on the diffusion and the reactions provoked by this writing during the convulse years of 1820-1821 in the Reign of the Two Sicilys, centered on the need for press freedom. The Galluppi text was written on behalf of the law regulating this freedom inescapable for the contruction of a liberal state and for the emergence of public opinion. Also, the writing mobilizes the censure, in its double role as repressing and punitive stake, while liberalism failed yet to be confirmed and absolutism renounced to disappear.
\end{abstract}

KEYWORDS: Naples, Sicily, press freedom, censure, public opinion, Ferdinand I of Borbon.

\section{RÉSUMÉ}

La diffusion et les réactions issues de la parution d'un écrit dans les années turbulentes de 18201821 pour le Royaume des Deux-Siciles, où l'on met en évidence la nécessité de la liberté de la presse sont le but de ce travail : l'opuscule de P. Galluppi est son leitmotiv. Ce texte a été préparé en vue de la loi régissant cette liberté indispensable pour la construction de l'état libéral et l'émergence de l'opinion publique, tandis que ledit écrit met en evidénce le travail de la censure dans son double rôle de répression et punition, à une époque où le libéralisme n'avait pas fini de se confirmer et l'absolutisme refusait encore de partir.

MOTS CLÉ: Naples, Sicile, liberté d'expression, liberté de la presse, censure, opinion publique, Ferdinand I de Bourbon. 
SUMARIO: 1. Una breve premessa. 2. L'Opuscolo su la libertà della stampa di Pasquale Galluppi. 3. Una conclusione.

\section{Una breve premessa}

Mentre si susseguivano dal 1816 al 1820 progetti e tentativi di rivoluzione sia a Napoli che in Sicilia ${ }^{1}$, il re di Spagna suggeriva a Ferdinando I, re delle Due Sicilie, di adottare la carta costituzionale di Cadice per accreditare il proprio diritto ad un'eventuale successione alla Corona madrilena ${ }^{2}$.

Gli echi di Cadice, alimentando i propositi d'insurrezione coltivati dalla Carboneria, riunivano insieme «l'impronta cattolica (che sembrerebbe manifestarsi al meglio nella cattolica constitución di Cadice), l'opzione monarchica (decisamente vincolante sul piano del progetto politico e fatale alla rivoluzione su quello degli avvenimenti storici) e l'aspirazione costituzionale (che prende[va] irrimediabilmente la forma della Carta spagnola, simbolo in quel momento degli ideali liberali) $»^{3}$.

Come sottolinea Girolamo Addeo, «i proclami, pubblicati a ritmo incalzante, si presentavano come autentiche petizioni: supplicando e ricordando al Re che aveva promesso una Costituzione, non risparmiavano aspri rimproveri e pericolose insinuazioni» ${ }^{4}$.

Era in tale contesto politico che, non a caso, il dibattito sulla libertà di stampa si faceva più acceso.

${ }^{1}$ Secondo Giorgio Spini, i moti del 1820-21 avevano una chiara fisionomia politica e sociale. Essi, a suo dire, rappresentavano «l'insurrezione della borghesia provinciale, affiliata alla carboneria ed attraverso questa in contatto con elementi della bassa ufficialità dell'esercito, che si impone con il suo colpo di mano sulle forze dello stato e sulla capitale. Nel suo trionfo trascina con sé l'idea della Costituzione di Spagna, cioé della costituzione democratica, priva di considerazione per i privilegi nobiliari, e riesce ad imporre anche questa alle sfere dirigenti della capitale che, per sorpresa, non fanno a tempo ad esprimere quella renitenza invincibile verso la democrazia, carbonismo, Costituzione di Cadice, che esse manifesteranno in modo sempre più deciso col passare del tempo, fino a determinare colla propria volontà negativa il crollo del regime costituzionale davanti all'avanzata austriaca» (G. Spini, Mito e realtà della Spagna nelle rivoluzioni italiane del 1820-21, Roma, 1950, p. 27).

${ }^{2}$ Cortese, La prima rivoluzione separatista siciliana 1820- 1821, Napoli, 1960, p. 16; G. Coniglio, I Borboni di Napoli, Milano, 1983, pp. 59-60.

${ }^{3} \mathrm{P}$. Colombo, Costituzione come ideologia. Le rivoluzioni italiane del 1820- 21 e la costituzione di Cadice, in Portello Valdés J. M., La Nazione cattolica. Cadice 1812: una costituzione per la Spagna (a cura di R. Martucci), Roma 1998, pp. 133-134. In particolare scrive Colombo: «la costituzione spagnola è innanzi tutto una costituzione monarchica: non per nulla, nel suo ispirarsi alla constitution francese del 3 settembre 1791 sta, sul piano costituzionale, il legame più diretto dei moti italiani con l'esperienza rivoluzionaria d'oltrealpe. Né va dimenticata, quale giustificazione dell'adozione napoletana di Cadice, la prossimità tra i due rami della stessa dinastia, regnanti rispettivamente in Spagna e nelle Due Sicilie» (Colombo, Costituzione come ideologia, pp. 145-146).

${ }^{4}$ G. Addeo, La libertà di stampa nel nonimestre costituzionale a Napoli, in Archivio Storico per le Province Napoletana, Napoli, 1989, p. 347. 
Il 16 agosto del 1815 veniva ripristinata la censura in vigore in Sicilia fino al $1806^{5}$. Qualche mese dopo, il 28 novembre, si disponeva che per tutti i libri —sia per quelli stampati nel Regno sia per quelli provenienti dall'estero- il controllo fosse affidato congiuntamente al Ministero dell'Interno, incaricato della pubblica istruzione, e al Ministero di Polizia, che aveva la facoltà di impedirne la pubblicazione o la circolazione. Al Ministero di Polizia, in particolare, era attribuita la competenza sugli opuscoli di pochi fogli, le opere periodiche e, più in generale, su tutte le carte volanti ${ }^{6}$.

Il sistema di controllo, nella pratica, era piuttosto complicato: il revisore esaminava l'opera e faceva rapporto all'incaricato provvisorio di polizia che, a sua volta, ne autorizzava la stampa. I tipografi, dal canto loro, erano obbligati a consegnare una copia dei libri che stampavano al dipartimento dell'Interno e un'altra al dipartimento di Giustizia, dove un altro ufficiale analizzava nuovamente il testo al fine di evitare elementi tali da «urtare con qualche disposizione o particolare veduta del governo ed ignota ai revisori ${ }^{7}$.

Con il Concordato del 1818 tra Ferdinando I e Pio VII si fissavano i diritti dello Stato e della Chiesa sulla stampa. L'art. 24 del medesimo Concordato disponeva che «ogni qualvolta gli Arcivescovi ed i Vescovi ne' libri introdotti, o che s'introducono, stampati, o che si stampano nel Regno, troveranno qualche cosa contraria alla dottrina della Chiesa, ed ai buoni costumi, il Governo non ne permetterà la divulgazione» ${ }^{8}$.

${ }^{5}$ F. Scaduto, Stato e Chiesa nelle Due Sicilie, con introduzione di A. C. Jemolo, Palermo 1969 ( $1^{a}$ edizione 1887), vol. I, pp. 351-352. Si veda, inoltre: S. Bottari, Stampa e censura in Sicilia nell'età del Risorgimento, in Potere e circolazione delle idee. Stampa, accademie e censura nel Risorgimento italiano, Atti del Convegno di studi nel bicentenario della nascita di Giuseppe Mazzini, a cura di D. M. Bruni e con prefazione di N. Graziani, Milano, 2007, pp. 377-408.

${ }^{6}$ Collezione delle leggi e de' decreti reali del Regno delle Due Sicilie, anno 1815, Napoli, pp. 518-520.

${ }^{7}$ Palermo, Archivio di Stato, Ministero e Segreteria di Stato presso il luogotenente generale. Ripartimento Polizia, busta 5, doc. 602, Napoli, 15 gennaio 1820.

${ }^{8}$ Collezione degli atti emanati dopo la pubblicazione del Concordato dell'anno 1818, Napoli 1829- 1854, vol. I, p. 13 e Giornale dell'Intendenza di Palermo, n. III, 24 aprile 1818, pp. 33-41. Con l'art. 20 del Concordato, l'autorità ecclesiastica otteneva, inoltre, senza previo esame dell'autorità laica, la pubblicazione delle pastorali ed istruzioni degli Ordinari diocesani : «Ai medesimi Arcivescovi e Vescovi sarà libero di comunicare col Clero e col popolo Diocesano per doveri dell'uffizio pastorale e pubblicare liberamente le loro istruzioni ed ordinanzioni sulle cose Ecclesiastiche». Giuseppe Bianco era stato molto polemico su questo Concordato che «induceva a credere prossima l'abolizione del Tribunale della monarchia ed apostolica legazia». Inoltre, scriveva: «il progresso dell'Isola veniva d'un tratto arrestato e appariva spaventevole lo spettro della barbarie. I buoni inorridirono alla pubblicazione del Concordato; i saggi fremettero; tutti tacquero sbigottiti di tanto servilismo verso il governo teocratico... le disposizioni del Concordato facevano retrogradare la società almeno di quattro secoli, attribuendo ai preti la dittatura sulla pubblica istruzione, istituendo nell'Isola tante nuove sedi inutili, lasciando guazzare taluni vescovi in una abbondanza di tesori. Inoltre, la restituzione dei beni confiscati e non alienati, la ripristinazione dei monaci e delle monache possidenti, l'accrescimento dei monaci mendicanti coi loro generali residenti a Roma erano fatali all'agricoltura, alle arti, alla popolazione, alle finanze dell'Isola, 
La censura preventiva ecclesiastica stabilita nel 1818 , sia sui libri prodotti nel Regno sia su quelli stampati all'estero, veniva abolita nel luglio del 1820, quando Ferdinando I, in conseguenza dei movimenti animati dai carbonari, era costretto a concedere per il Regno di Napoli la Costituzione di Cadice'.

Per effetto dell'art. 371 della nuova «carta» si riconosceva a tutti la «libertà di scrivere, stampare e pubblicare le loro idee politiche senza bisogno di anteriore revisione, o approvazione, o licenza; ma per altro sotto le limitazioni e responsabilità da stabilirsi dalle leggi» ${ }^{10}$.

Tale libertà, tuttavia, doveva essere opportunamente disciplinata. La Giunta provvisoria di Governo predisponeva così un progetto di decreto da sottoporre all'approvazione sovrana del vicario Francesco, il quale, da subito manifestava il fermo convincimento di non limitare preventivamente i cittadini nell'esprimere con la stampa le proprie idee, ma, eventualmente, solo di reprimere eventuali abusi.

Si stabilivano così quattro principi cui fare riferimento per la stesura del progetto, ovvero ci si basava sull'assunto che la libertà di stampa fosse «incompatibile con misure preventive; i pericoli conseguenti agli abusi di essa [fossero] da preferirsi a quello di bloccare le parole tra le labbra del cittadino; i delitti commessi con la stampa non [fossero] diversi da quelli consumati con altri mezzi di comunicazione e come tali [fossero] da punire, e non da prevenire; essi, come delitti d'opinione, dovevano essere salvaguardati da ogni sospetto di prevenzione e di prevaricazione e giudicati, pertanto, da un giury, oltre che imparziale, indipendente da ogni influenza del governo» ${ }^{11}$.

Nel preambolo del testo da sottoporre all'approvazione sovrana si richiamava espressamente l'art. 371 della Costituzione gaditana e si sottolineava come la libertà di stampa «indirizzata al fine d'istruire i cittadini nei loro diritti e di prevenire o mettere al giorno gli abusi di autorità che avessero potuto offendere la Costituzione» non dovesse essere utilizzata per offendere la religione e la morale pubblica, e soprattutto per indurre il popolo alla rivoluzione ${ }^{12}$.

$\mathrm{Si}$ vietava, di conseguenza, la diffusione di proclami e stampe senza il preventivo permesso delle autorità di Pubblica Sicurezza; di pubblicare opere tendenti a promuovere l'insurrezione popolare, o contrarie alla religione cattolica e lesive dell'ordine pubblico (artt. 4-7). Si disponeva che la censura repressiva fosse attuata dalla Giunta provvisoria a tutela della libertà di stampa, nominata dal Re e composta in ogni provincia da sei membri scelti tra gli accademici più illustri del Regno (art. 10).

perché molti prodotti passavano nella classe dei consumatori, dei poltroni e dei parassiti»(G. Bianco, La rivoluzione siciliana del 1820, Firenze, 1905, pp. 10-11).

${ }^{9} \mathrm{Si}$ veda, in particolare: W. Maturi, Il Concordato del 1818 tra la Santa Sede e le Due Sicilie, Firenze, 1929.

${ }^{10}$ Collezioni delle leggi e Decreti reali del Regno di Napoli, anno 1820, num. 1, pp. 4-6.

${ }^{11}$ Addeo, La libertà di stampa, p. 359.

${ }^{12}$ Addeo, La libertà di stampa, p. 360. 
Era, quindi, proprio alla Giunta che gli organi di pubblica sicurezza, a seguito di un primo esame dell'opera giudicata «sospetta» o «pericolosa», dovevano rivolgersi. La Giunta, a sua volta, dopo avere intimato l'autore dello scritto a presentare delle «giustificazioni», era tenuta ad esprimere il suo giudizio e a sancire gli estremi dell'accusa (art. 11).

L'autore, entro venti giorni dalla notifica della stessa, aveva facoltà di appellarsi alle Università degli Studi (di Napoli, Palermo o Catania) competenti per territorio, che potevano smentire le imputazioni (art. 12).

Per i libri provenienti dall'estero era, invece, richiesto un visto d'ingresso, subordinato al parere della stessa Giunta, che doveva essere rilasciato entro ventiquattro (art. 16). In caso di parere negativo, questo doveva essere motivato e passava al vaglio del Ministero dell'Interno, quale suprema autorità di controllo.

La censura preventiva, di fatto, rimaneva in vigore solo per le opere in materia teologica ed era affidata, non più ai vescovi, ma alle facoltà di teologia delle Università (art. 15). La formulazione del decreto normativo sulla pubblicazione di scritti di materia religiosa rappresentava il momento più difficile del dibattito costituzionale, dal momento che, invece, le Cortes spagnole avevano accettato la censura preventiva dei Vescovi ${ }^{13}$. «E' fuori di dubbio»-sostiene Domenico Rodia - «che la legislazione del' 20 sulla stampa segna un altro passo avanti sulla via del laicismo perché la censura preventiva, conservata solamente per i libri che ex professo si occupano di questioni teleologiche, viene affidata alle Facoltà teologiche competenti e non ai Vescovi» ${ }^{14}$.

Tale progetto veniva approvato il 26 luglio del 1820 ottenendo il parere favorevole della Giunta, del governo e anche la sanzione sovrana ${ }^{15}$. Non mancavano, tuttavia, le polemiche.

\section{L'Opuscolo su la libertà della stampa di Pasquale Galluppi}

Al fine di ricostruire il nesso che collega la libertà di stampa e l'impegno politico di molti patrioti nella formazione dell'opinione pubblica, nonché per

13 Si veda Decreto «Sopra la libertà politica della stampa in Spagna», in Giornale Costituzionale del Regno delle Due Sicilie, XVIII, venerdì 28 luglio 1820, pp. 75-76.

${ }^{14}$ D. Rodia, La censura sulla stampa nel Regno delle Due Sicilie dal 1815 al 1848, estr. da Samnium, Anno XXX, n. 1-2 gennaio-giugno 1957, p. 80.

${ }^{15}$ Manifesto della Giunta provvisoria di governo del Parlamento Nazionale, [Napoli, 1820]. La legge veniva riportata dal giornale L'Imparziale n. 10 del 29 agosto 1820. In Sicilia - faceva notare Niccolò Palmieri- «tutti gli stampatori di Palermo furono chiamati in casa del presidente della Gran Corte, ove trovossi l'avvocato fiscale, ed ambi i magistrati dichiararono loro che se alcuno di essi ardiva di stampare qual si fosse carta senza il permesso del governo, sarebbe severamente punito; e come uno di quei miseri ebbe appena nominata la costituzione, gli saltarono addosso i due magistrati, minacciandolo che ad onta di centomila costituzioni gli avrebbero mandati in galera» (N. Palmeri, Saggio storico e politico sulla Costituzione del Regno di Sicilia infino al 1816 con un'appendice sulla rivoluzione del 1820, con una introduzione e annotazioni di anonimo [Michele Amari], Losanna, 1847, p. 232). 
ricavare talune chiavi di lettura per meglio comprendere il contesto storicoistituzionale del Regno, ho voluto prendere in esame un opuscolo stampato a Messina nel 1820 presso Antonio D'Amico Arena che esamina la legge del 26 luglio su «la libertà individuale del cittadino» ${ }^{16}$.

Tale libretto, di complessive 59 pagine e suddiviso in 3 capitoli, rispettivamente: I. Della Libertà in generale; II. Della libertà di pensare; III. Della libertà della stampa, era attribuito all' "autore del Saggio filosofico su la critica della conoscenza», pubblicato a Napoli nel 1819, ovvero al barone Pasquale Galluppi ${ }^{17}$.

Questi, nato a Tropea nel 1770 dal matrimonio fra i cugini Vincenzo e Lucrezia Galluppi, rispettivamente il ramo siciliano e calabrese della medesima famiglia ${ }^{18}$, non potendo contare su cospicue rendite fondiarie, era stato avviato agli studi seminarili presso la città di Messina, dove, pubblicava gli Opuscoli filosofici, probabilmente collegati con i precedenti, ma inediti, Pensieri filosofici sulla libertà individuale compatibile con qualunque forma di governo ${ }^{19}$.

${ }^{16}$ Opuscoli filosofici su la libertà individuale del cittadino. Opuscolo in cui si esamina la legge provvisoria de' 26 luglio 1820 su la libertà della stampa dell'autore del Saggio filosofico su la critica della conoscenza, Messina, 1820.

${ }^{17}$ Su Pasquale Galluppi si veda: Napoli, Archivio di Stato, Archivi privati: Galluppi di Cirella; Restaurazione: Pubblica Istruzione, f. 280; Napoli, Biblioteca nazionale, Sezione mss: cfr. P. Franzesa, Opere mss. ed a stampa di P. G. nella Bibl. Nazionale di Napoli. Catalogo ragionato, Napoli, 1987; P. E. Tulelli, Intorno alla dottrina ed alla vita politica del barone Pasquale Galluppi. Notizie ricavate da alcuni suoi scritti inediti e rari, in Atti della Reale accademia di scienze morali e politiche di Napoli, II, 1865, pp. 101-121; Id., Sopra gli scritti inediti del barone P. G., in Atti dell'Acc. di scienze morali e politiche di Napoli, III, 1867, pp. 81-131; Autobiografia (15 ag. 1822), a cura di F. Pietropaolo, in Riv. di filosofia scientifica, VI, 1887, pp. 260- 265; N. Arnone, Pasquale Galluppi giacobino?, in Studi dedicati a Francesco Torraca nel XXXVI anniversario della sua laurea, Napoli, 1912, pp. 129-152; G. Gentile, P. G. giacobino?, in Rassegna di Storia del Risorgimento, II, 1914, pp. 389 e ss.; A. Sofia, Galluppi e Pancaldo: due patrioti dalla vecchia alla nuova Italia, Messina, 1988; M. Di Napoli, Galluppi Pasquale, in Dizionario biografico degli italiani, Istituto della Enciclopedia Italiana, 51 ${ }^{\circ}$ vol., Roma, 1998, pp. 748-751; Gli «Elementi di filosofia» di Pasquale Galluppi. Fra ragiona teoretica e metodologia storica, Atti del V Convegno di studi galluppiani, Tropea-Drapia, 2003, a cura di S. Venezia, Soveria Mannelli, 2007, e, in particolare, L. Meligrana, Pasquale Galluppi e la società tropeana tra Sette e Ottocento. Vicende personali e giudizio storico-politico, pp. 43-65.

${ }^{18}$ Il barone Galluppi scriveva nella sua Autobiografia: «I miei genitori furono il Barone don Vincenzo e donna Lucrezia Galluppi, tutti e due della stessa famiglia Galluppi, una delle antiche famiglie patrizie della città di Tropea» (C. Toraldo Tranfo, Saggio sulla filosofia del Galluppi, Napoli, 1902, p. 29). Anche Vincenzo Lastrucci riferiva: «Pasquale Galluppi, unico figlio del Barone D. Vincenzo di S. Lucia del Mela, città poco distante da Messina, e di Lucrezia della stessa famiglia, nacque a Tropea in Calabria il 2 aprile 1770, un anno dopo la morte del Genovesi» (Pasquale Galluppi, Studio Critico, Firenze, 1890, pp. 102-103).

${ }^{19}$ Toraldo Tranfo, Saggio sulla filosofia, pp. 106- 107. A Messina tra il 1820 e il 1827 erano pubblicati gli Elementi di filosofia, di 6 tomi; nel 1822 e nel 1832 quattro tomi del Saggio filosofico sulla critica della conoscenza (i primi tomi erano usciti a Napoli nel 1819); nel 1827 le Lettere filosofiche sulle vicende della filosofia relativamente ai principii delle conoscenze umane da Cartesio sino a Kant inclusivamente, ripubblicate a Napoli nel 1838 e indirizzate al canonico G. Fazzari, 
Desta qualche meraviglia che le opere del barone siano sfuggite alla repressione successiva e che il Galluppi, nel 1806 semplice dipendente del Fisco per il distretto di Tropea, nonostante le ripetute richieste (disattese) di essere chiamato come sottointendente a Reggio Calabria nel $1812^{20}$, sia stato nominato soltanto nel 1831, ormai sessantenne, professore nell'Università napoletana, dal marchese di Pietracatella, Giuseppe Ceva Grimaldi, ministro dell'Interno che però faceva capo anche all' Istruzione, cui peraltro dedicherà l'opera Filosofia della volontà (1832-40 ${ }^{21}$.

Come scriveva Francesco Fiorentino «le novità politiche ne avevano eccitato l'anima; ma con l'intervento austriaco troncata ogni speranza sul nascere, dovette rassegnarsi, e tacere; e adoperarsi a far dimenticare i sensi liberali testé palesati» ${ }^{22}$.

L'ostilità ecclesiastica, a seguito di una denuncia anonima che coinvolgeva il suo maestro Santacolomba (noto per le simpatie gianseniste) ${ }^{23}$ e l'adesione alla causa liberale duranti i moti del 1820 avevano pesato sulla sua fortuna almeno fino al 1831, anno in cui si riavvicinava alla monarchia borbonica e dedicava, quasi a voler far dimenticare i suoi trascorsi, un sonetto e un discorso accademico a Ferdinando II all'indomani della sua incoronazione.

Divenuto membro delle più prestigiose accademie locali (Sebezia e Pontiniana), il Galluppi nel 1836 veniva chiamato da mons. G. M. Mazzetti a far parte della Giunta della Pubblica Istruzione e il suo pensiero e la sua fama si diffondevano tanto celermente da ottenere prestigiosi riconoscimenti, quali la nomina a socio corrispondente straniero dell'Accademia di Francia nel 1838 e l'onorificenza della Legion d'onore nel $1841^{24}$.

L'opuscolo preso qui in esame risulta di particolare interesse perché, oltre a documentare un momento travagliato della storia costituzionale dell'isola, anticipando il dibattito parlamentare sulla regolamentazione della libertà di stampa che interesserà il '48 siciliano, è l'unico scritto politico a noi pervenuto e fortunosamente ritrovato del Galluppi.

Come scrive il Toraldo, «la sua vita non ha vicende politiche, e, se qualche volta compose qualche scritto di sensi liberali, s'avvide subito delle conseguenze possibili, e cercò ben tosto di dimenticarlo e di farlo dimenticare» ${ }^{25}$.

docente di filosofia presso il seminario di Tropea (Di Napoli, Dizionario biografico degli Italiani, pp. 748-751).

${ }^{20}$ Il 15 agosto 1822, Pasquale Galluppi nella sua Autobiografia scriveva: «Non ho avuto altra carica se non quella di controloro delle contribuzioni dirette, dallo stabilimento della fondiaria sin'oggi, per lo spazio di anni 17» (Toraldo Tranfo nel Saggio sulla Filosofia, pp. 29-32).

${ }^{21}$ Luigi Settembrini, che fu allievo del Galluppi, ci tramanda notizie del maestro nelle sue Ricordanze della mia vita, Napoli, 1879, p. 53.

${ }^{22}$ F. Fiorentino, Storia della Filosofia, Napoli 1887, p. 608.

${ }^{23}$ A pesare sulla sua riputazione era soprattutto il giudizio di Mele, vescovo di Tropea: «non gode buona fama, perché si pretende aversi ingoiato collo studio vari errori della vana filosofia» (E. Di Carlo, Una denunzia anonima contro P. G., in Archivio storico per la Calabria e la Lucania, VII [1937], pp. 35-40).

${ }^{24}$ F. Barritta, I personaggi di Tropea e dintorni, Tricase, 2014, p. 48.

${ }^{25}$ Toraldo Tranfo, Saggio sulla Filosofia, p. 28. 
Veniamo a conoscenza di altri scritti politici del Galluppi da Francesco Guardione, il quale riferisce in particolare di due opuscoli: Sulla libertà di stampa e Sulla libertà di coscienza, entrambi del 1820 e «rarissimi... perché venuti meno gli ordini costituzionali, la prudenza consigliò quasi la soppressione; né alcuno poté mai darci contezza del primo, perché soltanto se ne fece cenno del titolo; né le più accurate ricerche, fatte in Messina, ... accrebbero le nostre speranze di poterlo rinvenire ${ }^{26}$. Del resto - faceva notare sempre Guardione- anche dopo la morte del barone nel 1846 «tempo chiuso pe' rigori di governo alle notizie e al discutere di vicende politiche, non fu concesso a' biografi e a' panegiristi di potere liberamente intrattenersi del cittadino e delle professate opinioni politiche, che si compiaceva inculcare con sensi di alta filosofia» ${ }^{27}$.

Si è voluto qui analizzare uno scritto emblematico di Pasquale Galluppi sulla libertà di stampa, che nel 1820 assumeva pregnante attualità e alimentava un ampio e sentito dibattito. Veniva, infatti, discussa — anche con particolare animosità- la legge del 26 luglio con l'intento di far maturare una vera e propria coscienza «nazionale» e promuovere la riflessione politica e l'educazione costituzionale del popolo. Proprio in quegli anni, con l'apprestarsi sia dei moti carbonari sia del cosidetto «nonimestre rivoluzionario», durante i quali sembrò profilarsi un liberalismo monarchico-costituzionale, riaffiorava l'impegno politico e civile del barone, fedele alle proprie convinzioni liberali.

L'autore, partendo dall'assunto che tale legge fosse lesiva del progresso civile, era contrario a qualsiasi limitazione che potesse apparire un attentato ai diritti conquistati con la rivoluzione per il timore che con la libertà di stampa si potesse limitare anche la libertà di opinione. La legge avrebbe, quindi, solo dovuto reprimere e punire gli eccessi ma non moderare la libertà di pensiero.

Come espressamente affermava l'autore, «il cittadino dee godere di una perfetta libertà per tutte quelle azioni, che non offendono i diritti particolari di ciascun uomo, né son contrarie al bene generale della società» ${ }^{28}$. Dal che seguiva che tutti, in qualunque forma di governo, dovrebbero godere della libertà di pensare e, quindi, anche di stampa.

Ci si soffermava, tuttavia, non tanto sulle azioni dei privati cittadini quanto, piuttosto, su quelle dei ministri e dei funzionari pubblici, i quali «appena investiti del potere, contraggono l'abito di riguardarlo come un patrimonio, di cui godono non per interesse generale, ma pel loro interesse particolare; non mica per la prosperità di tutti, ma pel loro vantaggio personale» ${ }^{29}$.

${ }^{26}$ F. Guardione, Due opuscoli di Pasquale Galluppi preceduti dallo studio critico «De’ concetti civili e politici apportati da Pasquale Galluppi nella rivoluzione del 1820», in Atti Accademia Peloritana, XXII, 1906, pp. 165-166.

${ }^{27}$ Ivi, p. 165.

${ }^{28}$ Opuscoli filosofici su la libertà individuale del cittadino. Opuscolo in cui si esamina la legge provvisoria de' 26 luglio 1820 su la libertà della stampa dell'autore del Saggio filosofico su la critica della conoscenza, Messina, 1820, p. 10.

${ }^{29}$ Ivi, p. 27. 
Anticipando il rapporto del 5 dicembre del 1820 in cui l'Intendente e il Procuratore Generale della Gran Corte Criminale di Trapani si lamentavano con il Ministro di Grazia e Giustizia degli attacchi apparsi nei giornali contro pubblici funzionari ${ }^{30}$, l'autore sosteneva che l'uomo virtuoso «che ha la passione del bene, e l'amore de' suoi doveri» non dovrebbe temere in alcun modo l'opinione pubblica, anzi «dee questo sacrifizio alla sua patria». Così, «l'uomo, che accetta un posto elevato dee sapere, che si espone alle tempeste, che chiama gli sguardi su di lui, che i rigori della censura perseguiteranno tutte le sue azioni» ${ }^{31}$.

Scopo della libertà di stampa doveva essere, quindi, proprio quello di svelare le manovre degli uomini in carica nell'espletamento delle loro funzioni, «di rischiarare la loro condotta, di smascherare i loro intrighi, di avvertire la società de' pericoli, che ella corre» ${ }^{32}$.

Del resto - si chiedeva l'autore - quale cittadino sarebbe mai così stolto da voler compromettere la sua stessa esistenza ed essere dichiarato calunniatore attaccando un ministro se non è convinto che questi sia colpevole e «tradisc[a] in segreto l'interesse del suo paese»? ? $^{33}$

Se ne evinceva che la libertà di stampa fosse al contempo «spavento de' tiranni e salvaguardia degli oppressi», favorevole agli uomini virtuosi, ma tuttavia funesta per quanti non seguano gli interessi generali ${ }^{34}$.

Ci si soffermava, quindi, in particolare sulla censura preliminare dei libri di religione, soggetti al controllo degli ordinari ecclesiastici. Secondo l'autore, la libertà di stampa sulle materie religiose avrebbe dovuto essere fondata su lo stesso diritto su cui era fondata la libertà di stampa nelle materie politiche, letterarie $\mathrm{e}$ filosofiche, poiché ciascuno avrebbe dovuto godere del diritto naturale e imprescrittibile d'istruire ed essere istruito anche nei dogmi religiosi ${ }^{35}$. Così si domandava: «un padre di famiglia non ha egli il diritto, ed il dovere d'istruire la sua

${ }^{30}$ Si legge nel rapporto, «convien mettervi un freno istallando colà la Giunta Protettrice onde ogni eccesso in genere di stampa possa essere sottoposto all'esame della medesima per darsi luogo al dovuto procedimento contro i colpevoli» (Napoli, Archivio di Stato, Ministero Interno. I Inventario, fasc. 48.33 della vecchia numerazione e fasc. 921 della nuova).

${ }^{31}$ Opuscoli filosofici su la libertà individuale del cittadino, p. 29.

${ }^{32}$ Continua l'autore sostenendo che questa libertà fosse «una sentinella vigilante, che giorno, e notte guarda lo stato, ella dà alcune volte de' falsi spaventi; ma un eccesso di prevedimento è preferibile ad una funesta sicurezza, e val meglio esser tuttora pronto a difendersi, sebbene il pericolo non sia sempre reale, che essere attaccati all'improvviso» (Ivi, pp. 27-28).

${ }^{33}$ Ivi, p. 28.

${ }^{34}$ Ivi, p. 30. Riprendendo la Costituzione francese del 1791 si vuole stabilire la responsabilità per le calunnie volontarie contro la proibità e la rettitudiine dei funzionari pubblici: «Le azioni de' particolari non appartengono al pubblico. La vita privata di un Cittadino è sua proprietà inviolabile, ed ogni attentato a questa proprietà è criminoso: e se pure il magistrato s'impadronisce d'un cittadino, non può egli pubblicare di sua vita privata, se non quel tanto, che alla causa strettamente appartiene. La stessa regola vale pe' pubblici funzionarj considerati come cittadini. Si mettano in libero esame i loro atti, ma si rispettino le loro intenzioni, e la loro vita privata» (Ivi, p. 34).

${ }^{35}$ Ivi, pp. 37-38. 
figliolanza nella Religione? Gli si potrebbe negare il diritto prezioso di farle conoscere il Vangelo di Gesù Cristo, da preservarla dal contagio dell'incredulità, e della superstizione?» ${ }^{36}$.

Era fondamentale, inoltre, distinguere la semplice manifestazione delle opinioni «irreligiose» dall'insegnamento delle stesse ${ }^{37}$, e successivamente operare un'altra distinzione tra «l'insegnamento fatto col fine di combattere, e distruggere la Religione Cattolica da un insegnamento, senza questo empio fine». Per attaccare la religione era necessario pubblicare delle proposizioni «contrarie ai dogmi religiosi, ed addurre de' raziocinj, e delle pruove per sostenerle» ${ }^{38}$, non potendosi confondere la semplice manifestazione delle opinioni contrarie alla religione cattolica con un reato.

Parimenti, pretendere che i sacerdoti prima di stampare un trattato di religione o una pastorale chiedessero il permesso della facoltà di teologia di Napoli, Catania o Palermo era «un attentare a' diritti originari della loro divina missione, un affidare il deposito della fede non all'Apostolato, ma a delle corporazioni umane, ancora introdurre il dispotismo teologico» ${ }^{39}$.

L'anno successivo, il 9 giugno del 1821, l'Arcivescovo di Napoli proponeva al Ministro di Stato degli Affari Ecclesiastici di «rimettere la Chiesa nel suo immemorabile diritto di esaminare i manoscritti prima di stamparsi per conoscere se si serbi illibata la dottrina e la Morale evangelica, di cui la sola Chiesa ne può discernere la verità, perché ad essa sola è affidato questo deposito». Il 14 luglio il Ministero dell'Interno, a cui era stato trasmesso dalla Segreteria degli Affari Ecclesiastici il memoriale dell'Arcivescovo, rispondeva che non era possibile contravvenire all'art. 24 del Concordato del 1818, secondo cui la censura preventiva spettava soltanto all'autorità laica e non era possibile «ripristinarsi l'antico sistema perché le opere da pubblicarsi fossero prima rivedute dalla Curia ${ }^{40}$. Norma ribadita, peraltro, anche nel 1828 a seguito della richiesta dei vescovi di esentarsi dal permesso del sovrano per la pubblicazione dei loro sinodi: il re dichiarava, infatti, che «non v'ha disposizione, la quale abbia annullato lo stabilimento generale, di non potersi i Sinodi Diocesani pubblicare, senza essere prima rassegnati a Sua Maestà, ed ottenuto il Sovrano permesso per la loro pubblicazione: stabilimento che la M.S. vuole esattamente osservare» ${ }^{41}$.

Differentemente dalla Spagna una legge che stabilisse la censura preliminare sui libri di religione non era, dunque, da ritenersi conveniente al Regno delle Due Sicilie: «Gli Spagnuoli vivevano sotto il giogo dell'inquisizione: un numero immenso di clero regolare gravitava sullo Stato: la rivoluzione distruggendo in

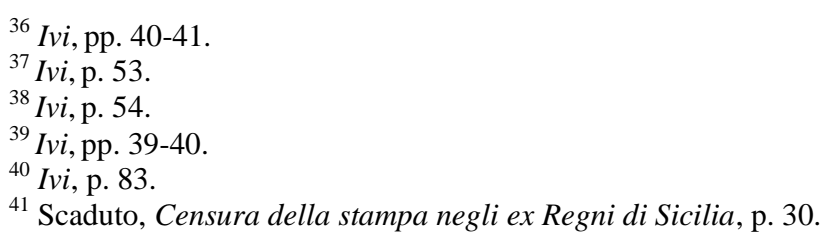


Ispagna, queste istituzioni, dovette, per non urtare violentemente pregiudizj troppo radicati, usare una certa circospezione sulla libertà di scrivere in materie religiose. Lo stato morale del nostro Regno è diverso. L'inquisizione non ha mai potuto germogliare in queste felici contrade» ${ }^{42}$.

Sempre nel 1820, a Napoli, l'abate Luigi Galanti, con cui l'autore dell'opuscolo in esame dichiara di essere in rapporti di amicizia ${ }^{43}$, nel Catechismo Costituzionale della monarchia per uso del Regno Unito delle Sicilie, polemizzava con i dettami della Costituzione spagnola che proibiva il pubblico esercizio di ogni altro culto al di fuori di quello cattolico, si soffermava sulla libertà di religione, «questione per noi del tutto oziosa, poiché avendo una sola religione non abbiamo bisogno di proclamare la libertà de'culti, come si è dovuto fare in altri paesi, che avevano seguaci di religioni diverse» ${ }^{44}$.

L'aggettivo pubblico, affiancato nella Costituzione all'esercizio della religione, era in questo caso per il Galanti «una garanzia contro ogni tentativo di tornare ai tempi nei quali si inquisiva contro le private coscienze», e si autorizzava la professione di altri culti in privato ${ }^{45}$.

La libertà di culto per l'abate non promuoveva «la ruina della religione», piuttosto «purifica[va] la religione, perché ne scaccia[va] i suoi veri nemici l'ipocrisia e la superstizione. Col fatto poi si osserva[va], che le varie sette ne'paesi, dove vi è tolleranza, prosperano tutte del pari» ${ }^{46}$.

Anche i Dialoghi politici fra due liberali, pubblicati a Napoli nel 1820, si concentravano sul tema della libertà di stampa e in sole 16 pagine riflettevano con un tono aspro il tentativo di far maturare una diversa coscienza politica nel Regno riportando un colloquio fra Il Prudente ed Il Riscaldato che ha per oggetto «la riforma di alcuni punti essenziali della nostra legislazione e della costituzione nel nostro regno adottata», in cui si danno anche «lezioni di moderazione necessaria per la felicità de' popoli» ${ }^{47}$.

${ }^{42}$ Opuscoli filosofici su la libertà individuale del cittadino, p. 44

${ }^{43}$ Ivi, pp. 56-57.

${ }^{44}$ L. Galanti, Catechismo Costituzionale della monarchia per uso del Regno Unito delle Sicilie, Napoli 1820, p. 21. Mi si consenta di rinviare a M. Basile, Sui «catechismi costituzionali» dell'avvocato Giovan Battista Nicolosi e dell'abate Luigi Galanti. Alcune brevi note, in Foro. Revista de Ciencias Jurídicas y Sociales Nueva Época, XVI-2, 2013, pp. 261-276 e M.A. Cocchiara, Catechismi politici nella Sicilia costituente (1812-1848), Milano, 2014.

${ }^{45}$ R. Lalli, Luigi Galanti al Parlamento napoletano del 1820, in Giuseppe Maria Galanti nella cultura del Settecento meridionale, Atti del convegno (Santa Croce del Sannio, 23-24 aprile), Napoli, 1984, p. 247

${ }^{46}$ Galanti, Catechismo Costituzionale della monarchia, p. 21.

${ }^{47}$ Dialoghi politici fra due liberali, Il Prudente ed Il Riscaldato di D. G. N., Napoli, 1820. Nel frontespizio, si legge che l'opuscolo «trovansi vendibile nella libreria del Sig. Raffaele Marotta largo S. Domenico maggiore n. 13 e 125, e nel deposito delle leggi al vico Afflitto a Toledo n. 23, a grana 4 il foglio». Mi si consenta di rinviare anche a M. Basile, Costituzionalismo e formazione dell'opinione pubblica in Sicilia (1812-1848), con prefazione di R. Martucci, Roma, 2016, pp. 96101. 
Con quest'opera, frutto non solo di una speculazione teorica ma anche di un'attenta analisi della realtà, i due interlocutori —il Prudente ed il Riscaldatointervenivano nella polemica, sottolineando come la libertà di stampa, senza proporre limitazioni preventive alla facoltà dei cittadini di esprimere le proprie idee, ma solo reprimere eventuali abusi, avesse come scopo principale quello di indottrinare i cittadini sopra i loro diritti e doveri e di reprimere tutti gli oltraggi contro la Costituzione, la religione e la morale pubblica, stabilendo, quindi, un nesso strettissimo tra la liberta individuale e il rispetto dei precetti costituzionali e religiosi.

Contrariamente agli articoli 231 e 571 della stessa costituzione spagnuola che consacravano la libertà politica della stampa «senza bisogno di anteriore revisione, o approvazione, o licenza» ${ }^{48}$, una legge del 26 luglio - sottolineava il Riscaldato - ne restringeva «l'uso, rendendo gli autori o gli stampatori risponsabili di ciò che scrivono o stampano, e creando delle giunte, dette protettrici, ma in effetto non sono che distruggitrici della libertà di stampa ${ }^{49}$

La legge del 26 agosto — scriveva anche Stefano Mazza - «merita[va] la riprovazione del Parlamento. Il divieto a qualunque cittadino di fare proclamazioni, di affiggere qualunque stampa, scritto o figura, senza il permesso del Magistrato della pubblica sicurezza, e la creazione di una Giunta Provvisoria per la revisione, tra l'altro dei libri, e delle stampe provenienti dall'estero attacca[va]no troppo da vicino l'esercizio di questa libertà. Censure precedenti alla stampa si oppon[eva]no alla Costituzione ${ }^{50}$.

Anche l'abate Giuseppe Rosselli, additando il decreto come anticostituzionale, scriveva una lettera di protesta contro la legge del 26 luglio e ne chiedeva la revoca, soprattutto per l'istituzione della Giunta — addebbitata dalla Carboneria all'inviso ministro dell'Interno Zurlo ${ }^{51}$ - di cui si cercava in tutti i modi d'impedirne l'esercizio ${ }^{52}$.

${ }^{48}$ Collezioni delle leggi e Decreti reali del Regno di Napoli, anno 1820, num. 1, pp. 4-6.

${ }^{49}$ Dialoghi politici fra due liberali, cit., p. 4. Si veda anche: Manifesto della Giunta provvisoria di governo del Parlamento Nazionale, [1820]. La legge era riportata dal giornale L'Imparziale, $\mathrm{n}$. 10, 29 agosto 1820 .

${ }^{50}$ S. Mazza, Memoria al Parlamento nazionale, s. 1., [1820], p. 12

${ }^{51}$ M. S. Corciulo, Prime esperienze costituzinali italiane: la rappresentanza politica di Terra d'Otranto al parlamento del 1820- '21, in Risorgimento Democrazia Mezzogiorno d'Italia, Studi in onore di Alfonso Scirocco, a cura di R. De Lorenzo, Milano 2003, pp. 507-523. Maria Sofia Corciulo faceva notare come la censura preventiva fosse una preoccupazione costante dei giornali dell'epoca. A tal proposito, gli Annali del Patriottismo il 28 luglio 1820 scrivevano: «Noi crediamo che in ogni governo liberale, anzi ragionevole, la libertà di stampa non debba avere altri limiti che quelli della responsabilità personale. Le ragioni che ci determinano non sono di giurisprudenza o di astrusa metafisica bensì della più facile intelligenza. Se la stampa va soggetta ai censori, siamo certi che il governo potrà e vorrà comprarli ed in conseguenza qualunque piccola restrizione distruggerà interamente la nobile prerogativa di esporre liberamente i propri pensieri. Diciamo la cosa come và: noi vogliamo scrivere contro tutti gli abusi e contro tutte le persone che non attendessero al loro dovere» (Id., Le istituzioni parlamentari in Francia (1815-16) Cento giorni, Seconda Restaurazione, Napoli, 1996).

${ }^{52}$ G. Rosselli, A.S.A.R. Il Principe Ereditario, Vicario Generale, 1820, p.3. L'abate Gioacchino Ventura il 26 settembre del 1820 scriveva un opuscolo, dedicato al principe ereditario, in cui negava le 
Peraltro, parrebbe potersi affermare che in molti avessero chiesto di essere esonerati dalla nomina tra i componenti della Giunta ${ }^{53}$, se la Giunta stessa, l'otto agosto, disponeva che fossero dichiarate «intollerabili le rinunce a tali funzioni da riguardarsi come dovere civico; [si] reclamava[no] energiche misure perché gli intendenti intimassero prontamente ai membri nominati per le giunte protettrici di prestare giuramento e perché si ponessero a disposizione degli stessi un ufficio con impiegati nel luogo dell'intendenza; perché si stabilissero turni di servizio nelle dogane, nonché norme di regolamento di procedura per la definizione del giudizio d'introduzione o meno, ovvero d'accusabilità» ${ }^{54}$.

Tuttavia, non ritenendo opportuno modificare il decreto del 26 luglio, il 28 agosto se ne integravano addirittura le norme, disponendo che tutti gli scritti pubblicati dovessero indicare il nome dell'autore e dell'editore con i relativi domicili nonché la tipografia, estendendo anche ai giornali e ai periodici le stesse disposizioni che prescrivevano al Ministero dell'Interno e di Grazia e Giustizia di informare della circolazione di scritti illeciti. Si statuivano, inoltre, pene per gli stampatori che avessero disatteso l'ordine di esibire gli scritti anonimi (specie se considerati «criminosi») innanzi agli ufficiali competenti ${ }^{55}$.

La libertà di stampa, come tutte le libertà, doveva avere dei limiti. Se la legge costituzionale decretava l'abolizione della censura, il Codice Penale per il Regno delle Due Sicilie, di contro, prescriveva pene severe per i trasgressori, in particolare per quanti avessero offeso la religione o il Governo con una pena che andava dal primo o secondo grado di prigionia sino all'esilio o l'ammenda correzionale (art. 314 e 315). Si disponeva, inoltre, per chi avesse insegnato, senza dolo, dogmi contrari alla religione l'interdizione dall'incarico ricoperto per uno o due anni, e per chi lo avesse fatto con «l'empio fine di distruggere o alterare» la religione, l'espulsione definitiva dal Regno (art. 100) ${ }^{56}$.

Si stabiliva altresì che quanti avessero pubblicato e affisso scritti o disegni non autorizzati dalla polizia (art. 316) fossero interdetti temporaneamente dai loro uffici

tesi sostenute da Rosselli. Respingeva la libertà politica della stampa come diritto di «esporre in faccia al popolo gli attentati commessi alla Costituzione», in quanto spettava unicamente al Parlamento e «non a i privati, occuparsi dei mezzi onde serbar illesi i dritti nazionali». Il popolo non godeva, infatti, dei mezzi per cambiare le leggi vigenti e «che se mai taluno credesse necessaria una interpretazione, o derogazione, o abrogazione rispetto ad alcuna legge; dovrebbe in questo caso diriggersi non al popolo, ma al corpo legislativo, cui tutta la nazione ha conferito il potere di bilanciare il bene comune col privato» (G.Ventura, Riflessioni Politiche sulla Libertà di Stampa, Napoli, 1820, pp. 4 e 11).

${ }^{53}$ Il vicepresidente della Giunta era il duca di Lusciano e gli altri membri il Ceva Grimaldi, il marchese di Pietracatella, Domenico Sarno, Gaetano Giannattasio, Nicola Fergola, Loreto Apruzzese, Salvatore M. Ronchi. Dal 12 settembre del 1822 la Giunta sarà, invece, composta dal Presidente dell'Università e da sei docenti universitari (Rodia, La censura nella stampa nel Regno delle Due Sicilie, pp. 82 e 84).

${ }^{54}$ Addeo, La libertà di stampa, p. 363.

${ }^{55}$ Ivi, pp. 365-366.

${ }^{56}$ F. Canofari, Commentario sulla Parte Seconda del Codice per lo Regno delle Due Sicilie, Napoli 1819, vol. I, pp. 23-28; pp. 37-44; pp. 62-66; pp. 187-194; pp. 267-269. 
(art. 317), comminando una pena dal primo al secondo grado di prigionia e un'ammenda o esilio correzionale (art. 312), in considerazione della gravità dell' offesa, della persona colpita, del luogo e delle conseguenze arrecate (art. 366) ${ }^{57}$.

Per i libelli famosi, ovvero per tutte quelle opere «pubblicate colle stampe in figure, in immagini, in incisioni, in emblemi, o in iscritto, o anche senza stampa quando sien pubblicate con pubblici affissi», si prescriveva una condanna dal primo al terzo grado di prigionia o di confine, più l'ammenda correzionale (art. 367) ${ }^{58}$.

Se ne evince come l'abuso della libertà di stampa dovesse essere punito penalmente, in particolare laddove con la stampa si offendevano la religione, «i diritti degli altri, ed i diritti del corpo sociale intero», quindi in tutti quei casi in cui si turbava «la pubblica tranquillità» e si promuoveva «la ribellione alle legittime autorità» ${ }^{59}$.

\section{Una conclusione}

In questo periodo, la censura sulla stampa assumeva una funzione per così dire «strategica» per sorvegliare e impedire la diffusione di testi propagatori di principi considerati «rivoluzionari». Non si traduceva solo in un problema di orientamento politico e di consenso ma in una vera e propria «azione di polizia» finalizzata al mantenimento dell'ordine pubblico.

${ }^{57}$ Si vietava a tutti i cittadini di far proclami o affissioni senza il permesso del Magistrato di Pubblica Sicurezza, tuttavia la parola «proclamazione» ha secondo l'autore un significato equivoco. «Certamente non può vietarsi il dirigere in una stampa qualunque il discorso a' cittadini, o a qualunque società, o classe di persone, il dare dei consigli utili, il fare dell'esortazioni. Può solo vietarsi il dar la forma pubblica, e legale a siffatti discorsi. L'affiggere, il pubblicare a suon di tromba, o di tamburo essendo una maniera pronta, ed efficace di far conoscere gli atti delle autorità costituite, sta bene che queste forme sieno riserbate a' pubblici funzionarj, e vietate a' particolari ... malgrado queste affermazioni... la facoltà di affiggere pubblicazioni di ogni sorta sta ben negata a' aprticolari, ove ciò venga eseguito negli stessi siti, in cui l'auotirtà pubblica affigge i suoi atti, ma in ogni altro perché impedirlo? Perché obbligare il librajo, che annuzia l'uscita di un'opera, l'impressario, che fa noto lo spettacolo della sera, il mercante, che avvisa l'arrivo delle sue merci, il dentista, che divulga la scoperta di un oppiato, il sacerdote, che fa noto il suo cambiamento di abiatazione, il paroco, che invita i fedeli al pio esercizio di qualche funzione religiosa, perché obbligarli in virtù della Costituzione, e della libertà della Stampa a domandare a' Magistrati un permesso incomodo, contrario lo direm francamente a' canoni d'ogni libero stato?» (Opuscoli filosofici su la libertà individuale del cittadino, pp. 50-52).

58 Francesco Canofari, commentando questo reato, sosteneva che «la ingiuria scritta e pubblicata colla stampa contiene una gravezza assai maggiore. Vero è che la scrittura manifesta nudamente il fatto; che lo rappresenta senza altra vivezza di quella che le sa dare lo scrittore; che la voce vi aggiunge e lo spirito e l'effetto; e che quindi la voce è assai più espressiva della scrittura. Ma non può riflettersi che la scrittura presenta qualche cosa di più permanente che la parola; che la parola si allontana per poco spazio, e non colpisce che le persone presenti; che la scrittura stampata si estende a persone, a luoghi, a tempi lontani. Non può non riflettersi che è dessa un de' mezzi più perfidi, più rapidi, più fecondi per la diffamazione; e che turba in modo tutto proprio la tranquillità pubblica e la individuale, forzando, a dir così, la vittima alla vendetta e talvolta allo spargimento del sangue» (Canofari, Commentario sulla Parte Seconda del Codice, vol. I, p. 191).

${ }_{59}$ Opuscoli filosofici su la libertà individuale del cittadino, p. 25. 
E' da notare come, in un momento in cui le istituzioni laiche e religiose erano passate da un'esigenza di controllo sulle pubblicazioni ad un utilizzo «pedagogico-educativo» delle stesse, l'azione repressiva fosse diretta non tanto ad una riforma della legge in vigore quanto al ripristino della censura preventiva, giudicata «antistorica» persino da molti conservatori ${ }^{60}$.

Prevenzione e repressione non si ponevano, pertanto, in alternativa l'una all'altra ma, piuttosto, in concomitanza. Tuttavia, mentre la censura repressiva poteva anche essere esercitata, rendendo - attraverso apposite norme e provvedimenti di polizia - stampatori e autori responsabili delle opere pubblicate, la prevenzione diveniva inefficace senza apposite norme repressive.

Come sottolinea Sandro Landi, la libertà di stampa era da intendersi come «una tendenza latente dei sistemi di censura a produrre -in circostanze di conflitto tra le giurisdizioni che esercita[va]no la censura- spazi di relativa tolleranza». Non si trattava, quindi, di una libertà che si poneva come alternativa alla censura, ma di una «libertà interstiziale, coestensiva alla censura, concepita e rivolta dal potere politico (o talvolta religioso) ai propri fini» ${ }^{61}$.

L'epilogo dei moti del '20-'21 è fin troppo noto, tuttavia ci si è chiesto quanto avesse contribuito la diffusione della stampa sulla capacità critica nei confronti dei poteri costituiti, se e in che misura, le norme di censura riuscirono ad arginare la pubblicazione di opere considerate «pericolose». Le disposizioni censorie s'inserivano, infatti, in un più vasto processo attraverso il quale i governi si dotavano di strumenti necessari per controllare l'opinione pubblica e sorvegliare la circolazione di idee ritenute «sovversive».

Bisogna chiedersi fino a che punto tali opuscoli riuscissero ad influire sull'opinione politica-istituzionale siciliana e quale fosse l'«eredità» da questi lasciata alla successiva rivoluzione del 1848. Il controllo e la repressione, senza dubbio, avevano influito sulle letture della popolazione ma i limiti di tali controlli offrivano - anche attraverso il contrabbando- possibilità di circolazione di idee.

Le pratiche censorie assumevano significati diversi nei vari periodi storici, divenendo - come scrive Maria Consiglia Napoli- nello stesso tempo, sistema «di controllo polizesco delle dissidenze politiche, sistema per indirizzare il consenso dell'opinione pubblica, strumento per la sorveglianza e la prevenzione di qualsiasi possibile devianza, specialmente nel campo della morale, per un popolo considerato allo stato infantile». Tuttavia, «queste varie prospettive non si manifestavano sempre con la stessa importanza nei diversi periodi. Di volta in volta prevaleva un aspetto sugli altri caratterizzando, così, con proprie peculiarità e specificità una determinata epoca storica ${ }^{62}$.

${ }^{60}$ M.I. Palazzolo, La perniciosa lettura. La Chiesa e la libertà di stampa nell'Italia liberale, Roma, 2010, pp. 28-29.

${ }^{61}$ G. Landi, Stampa, censura e opinione pubblica in età moderna, Bologna, 2011, p. 83.

${ }^{62}$ M.C. Napoli, Letture proibite. La censura dei libri nel Regno di Napoli in età borbonica, Milano, 2002, p. 8. 
Le principali disposizioni finalizzate a regolamentare la produzione, l'importazione e la commercializzazione dei vari scritti, in un'alternanza di fasi storiche che determinavano l«irrigidimento censorio» o la moderazione, imponevano un continuo riassetto amministrativo degli organi censori, della struttura burocratica poliziesca e degli stessi ruoli e funzioni dei revisori.

Il sistema di controllo, nella pratica, risultava piuttosto complicato e, comunque, inefficace. Vediamo, infatti, che, nonostante i vari progetti di riforma intesi a prevenire l'abuso della libertà di stampa e le modifiche in merito al dazio sui libri importati, cui si accompagnava la creazione di una Giunta protettrice della libertà di stampa, i patrioti continuavano ad esprimere «sempre o quasi sempre, e senza impacci» ${ }^{63}$ il loro pensiero.

${ }^{63}$ A. Galante Garrone, «I Giornali della Restaurazione (1815- 1847)», in La stampa italiana del Risorgimento, a cura di V. Castronuovo e N. Tranfaglia, Roma-Bari, 1979, p. 85. 